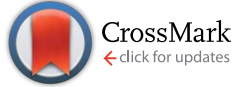

Cite this: RSC Adv., 2015, 5, 36969
Received 15th March 2015 Accepted 14th April 2015

DOI: $10.1039 / \mathrm{c} 5 \mathrm{ra0} 4558 \mathrm{~d}$

www.rsc.org/advances

\title{
Optimization of graphene-based materials outperforming host epoxy matrices $\uparrow$
}

\author{
L. Guadagno, ${ }^{\text {aa }}$ M. Raimondo, ${ }^{a}$ L. Vertuccio, ${ }^{a}$ M. Mauro, ${ }^{\text {b }}$ G. Guerra, ${ }^{\text {b }}$ K. Lafdi, ${ }^{c}$ B. De \\ Vivo, ${ }^{d}$ P. Lamberti, ${ }^{d}$ G. Spinellid and V. Tucci ${ }^{d}$
}

The degree of graphite exfoliation and edge-carboxylated layers can be controlled and balanced to design lightweight materials characterized by both low electrical percolation thresholds (EPT) and improved mechanical properties. So far, this challenging task has been undoubtedly very hard to achieve. The results presented in this paper highlight the effect of exfoliation degree and the role of edge-carboxylated graphite layers to give self-assembled structures embedded in the polymeric matrix. Graphene layers inside the matrix may serve as building blocks of complex systems that could outperform the host matrix. Improvements in electrical percolation and mechanical performance have been obtained by a synergic effect due to finely balancing the degree of exfoliation and the chemistry of graphene edges which favors the interfacial interaction between polymer and carbon layers. In particular, for epoxy-based resins including two partially exfoliated graphite samples, differing essentially in the content of carboxylated groups, the percolation threshold reduces from $3 \mathrm{wt} \%$ down to $0.3 \mathrm{wt} \%$, as the carboxylated group content increases up to $10 \mathrm{wt} \%$. Edge-carboxylated nanosheets also increase the nanofiller/epoxy matrix interaction, determining a relevant reinforcement in the elastic modulus.

\section{Introduction}

The use of graphite and/or exfoliated graphite (EG) has been extensively explored in order to produce conductive thermoplastic and/or thermosetting polymers with enhanced chemical and physical properties, ${ }^{1-7}$ but a clear understanding concerning the optimal transfer of the filler properties to the polymer composite has not yet been achieved. The efforts of material designers have been focused on the use of exfoliated graphite based polymer especially in the area of lightweight materials. These materials are strongly desired for better environmental and economic sustainability particularly in the transport sector, where future perspectives have to take into account the two main principles of minimizing vehicle weight, to deliver fuel/energy minimization; and compliance with sustainability requirements.

Polymer/graphene based nanocomposites show a transition from insulating to conducting behavior at the onset of a

${ }^{a}$ Department of Industrial Engineering, University of Salerno, Via Giovanni Paolo II 132, Fisciano (SA) 84084, Italy. E-mail: lguadagno@unisa.it; Tel: +39089964142 ${ }^{b}$ Department of Chemistry and Biology, University of Salerno, Via Giovanni Paolo II 132, Fisciano (SA), 84084, Italy

${ }^{\circ}$ University of Dayton, 300 College Park, Dayton Ohio, 45440, USA

${ }^{d}$ Department of Information Engineering, Electrical Engineering and Mathematics Applied University of Salerno, Via Giovanni Paolo II 132, Fisciano (SA), 84084, Italy $\dagger$ Electronic supplementary information (ESI) available. See DOI: 10.1039/c5ra04558d percolating structure with electrical percolation thresholds lower than 0.5 vol\% and high electrical conductivity $\left(\sim 0.1 \mathrm{~S} \mathrm{~m}^{-1}\right)$ associated with the homogeneous dispersion of fully exfoliated graphene samples..$^{8-18}$ Conversely, many researchers reported different data, ${ }^{19-21}$ i.e. even with an excellent graphene dispersion, materials with rather high percolation thresholds and poor electrical conductivities ${ }^{19-22}$ are obtained, probably due to the lack in forming interconnected conductive paths. The large improvement in electrical conductivity is generally attributed to several mechanisms, such as better graphite exfoliation, ${ }^{\mathbf{1 0}, 11,13-15,18}$ special graphene, ${ }^{8,9,12}$ polymer functionalization ${ }^{16}$ or to suitable grafting of polymers into graphene. ${ }^{16,17}$

The work here described demonstrates that full exfoliation of graphite (graphene layers) may not be the approach to pursue to achieve very low percolation thresholds and high electrical conductivity especially for bulk polymers. The presented results highlight that perfect graphene layers (without defects) originated from full exfoliated graphite tend to reassemble during the manufacturing of the nanocomposites. In fact, strong functionalization procedures are needed to obtain graphene in the form of single layer embedded in the polymeric matrix.

In this last case, the functional groups attached to the graphene layers prevent the re-assembling of layers due to steric and energy factors. Unfortunately, due to the transition from $\mathrm{sp}^{2}$ to $\mathrm{sp}^{3}$-hybridization of carbon atoms, functionalized single layers (SL) of graphene tend to lose delocalized electrons and, 
therefore, the very interesting electronic properties of graphene, hence reducing the electrical conductivity of graphene-based nanocomposites. This effect is further worsened by the difficulty of single layers to form conductive paths inside the bulk polymeric matrix due to characteristic morphological features of functionalized SL graphene layers. In fact, the functional groups act as defects imparting to the layers strong tendencies to screw up which lead to severe inhomogeneities in the nanofiller dispersion also preventing the formation of conductive pathways. On the other hand, significant benefits in terms of physical properties can be achieved for ultrathin graphitic stacks which preserve a large part of $\mathrm{sp}^{2}$-hydridized carbon atoms, and hence their graphene-like electronic properties. A deep balance and control of the inherent complexity of these systems at nanoscale level may drive the changes in the nanocomposite properties towards the set goals. In light of these considerations, samples of partially exfoliated graphite have been considered both to avoid the negative effect of single graphene layers and to maximize the beneficial effects of graphene-based materials for bulk samples.

The results here discussed seem to indicate that polymer (epoxy-based) composites with a very small percentage (starting from $0.1 \%$ ) of graphitic materials, exhibiting a large fraction (nearly $40 \%$ ) of highly ordered hexagonal graphite, can reach percolation thresholds and electrical conductivities similar and even better than those obtained for graphene-based nanocomposites.

Edge structures of graphitic blocks can be controlled for improving the performance of nanocomposites, as here evidenced by analyzing the properties of epoxy resins filled with very similar graphene-based materials, differing essentially for the exfoliation degree and consequently for the concentration of carboxylated groups on the nanoparticle edges. The impact of the chosen strategy on the electrical and mechanical properties is very significant. The chemistry of graphene edges strongly affects the physical properties of the resin where these nanoparticles are embedded and drive the changes in the nanocomposite properties towards the desired goals.

\section{Results and discussion}

\subsection{Characterization of the nanofiller}

Exfoliated graphite was prepared by processes of intercalation and exfoliation starting from high surface area of natural flake graphite with an average diameter of $500 \mu \mathrm{m}$. The intercalated graphite compound was subjected to sudden heat treatment in a high temperature reactor $\left(900{ }^{\circ} \mathrm{C}\right)$.

Changes in the degree of exfoliation were obtained by changing the resident time in the fluidized bed as described in the Experimental section. The change in the exfoliation degree also causes a different percentage of carboxylated groups on the nanoparticle edges. Here, to simplify the treatment, the results of two samples are shown. The comparison among different analyzed fillers is described in more detail in the section ESI. $\dagger$

In particular, two samples of partially exfoliated graphite (pEG) and carboxylated partially exfoliated graphite (CpEG), differing for the exfoliation degree and hence for the content of carboxylated groups, were prepared with the aim to achieve consistent comprehension about the properties of resins filled with graphene-based nanoparticles.

Fig. 1 presents (a) the TGA thermograms of pEG and CpEG samples (at heating rate of $10{ }^{\circ} \mathrm{C} \mathrm{min}^{-1}$ ) under air atmosphere, (b) the X-ray diffraction patterns, (c) the micro-Raman spectra recorded at the edge of the basal plane of the two sample and (d) the Scanning Electron Microscopy (SEM) images.

Fig. 1(a) shows that the pEG sample starts to lose weight at $650{ }^{\circ} \mathrm{C}$ due to the combustion to carbon dioxide. The CpEG powder exhibits two main steps of mass loss at 300 and $650{ }^{\circ} \mathrm{C}$, which can be attributed to the removal of oxygen-containing groups and carbon oxidation, respectively. TGA curves indicate an amount of carboxylated groups of about $10 \mathrm{wt} \%$ for the sample CpEG.

The X-ray patterns of Fig. 1(b) show an intense and narrow 002 graphite reflection, (with $d=0.339 \mathrm{~nm}$, and high correlation length perpendicular to the graphitic planes, close to 40 $\mathrm{nm}$ ) superimposed on a broad amorphous halo. The appearance of 101 and 112 reflections indicate the presence of hexagonal graphite with $\mathrm{ABAB}$ stacking of layers. The ratio between the area of the 002 peak and of the amorphous halo roughly centered at $2 \theta=26^{\circ}$ indicates that the percentage of exfoliated graphite is slightly different for the two samples (56\% for pEG and $60 \%$ for CpEG). Hence, pEG and CpEG samples, although markedly different as for their functionalization level, exhibit very similar structural organization. In particular, CpEG with respect to pEG shows a small increases in the amount of the exfoliated phase (7\%) and of surface area $(11 \%)$ (see, inset in Fig. 1(b)).

In the Micro-Raman (MR) spectra (Fig. 1(c)), the D band at $1351 \mathrm{~cm}^{-1}$ associated with the edge distortion ${ }^{23}$ is negligibly weak due to the presence of graphitic blocks in both samples with a ratio of the D-band to G-band intensity $\left(I_{\mathrm{D}} / I_{\mathrm{G}}\right)$ equal to 0.028 and 0.013 for pEG and CpEG samples respectively. The 2D peak between 2600 and $2800 \mathrm{~cm}^{-1}$ in bulk graphite consists of two components $2 \mathrm{D}_{1}$ and $2 \mathrm{D}_{2} \cdot{ }^{24}$ The relative intensity of these two peaks can be associated with the number of layers for graphene blocks containing less than 5 layers. In particular, it was noted from early studies that an increase in layers leads to a significant decrease of the relative intensity of the lower frequency $2 \mathrm{D}_{1}$ peak. ${ }^{25}$ Although pEG and CpEG samples contain graphitic blocks composed of a number of layers $>5$, this type of investigation was qualitatively applied to get further information from the MR spectra. In order to separate the individual peaks $2 \mathrm{D}_{1}$ and $2 \mathrm{D}_{2}$, a curve resolving algorithm was applied; ${ }^{26}$ the ratio $I_{2 \mathrm{D}_{1}} / I_{\mathrm{G}}$ is 0.226 and 0.251 for $\mathrm{pEG}$ and CpEG samples respectively. The small increase in the value of this ratio is in agreement with the RX investigation showing a small increase in the fraction of the exfoliated phase of CpEG with respect to pEG sample. These two spectra are very different from that detected for the completely exfoliated graphene SL (see ESI $\dagger$ ).

SEM investigation of pEG sample in Fig. 1(d) evidences in some zones (top right) structures like veils of silk on the flat surface of the pin stub; however in all zones flat flake-like surfaces can be observed. SEM image of the CpEG sample 
a)

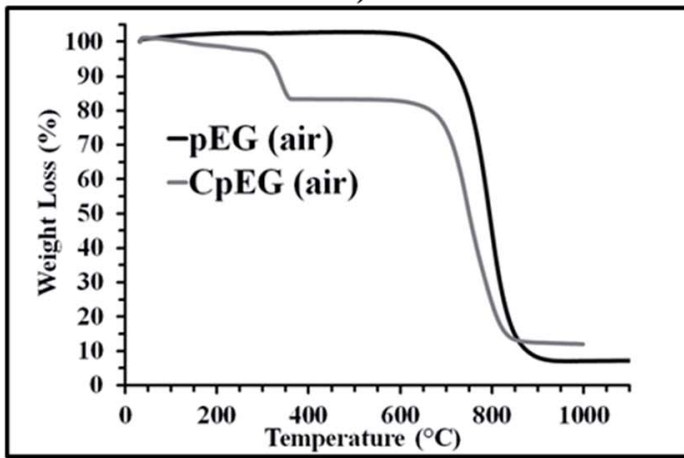

c)

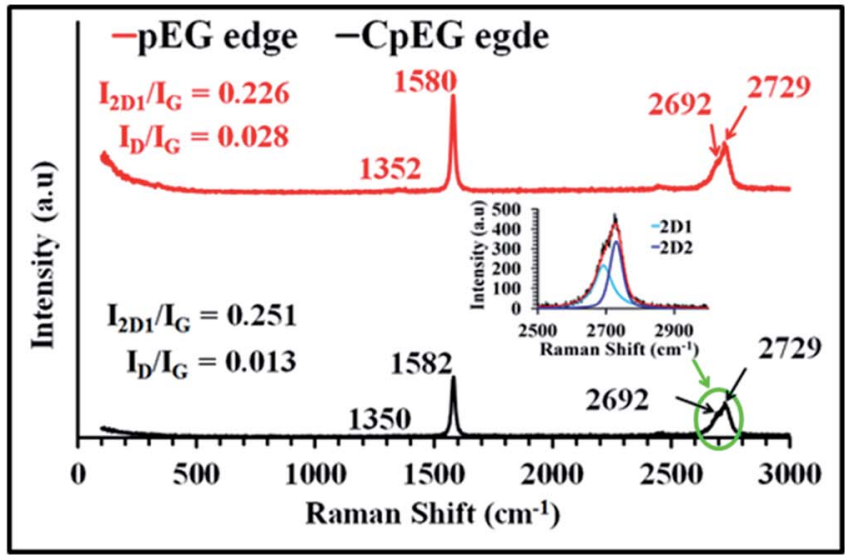

b)

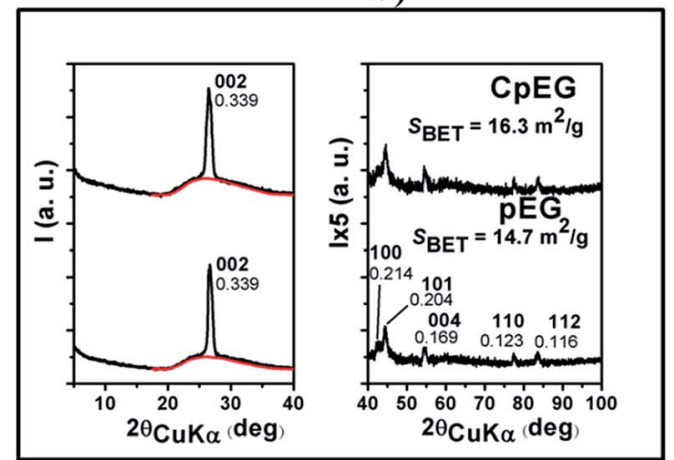

d)

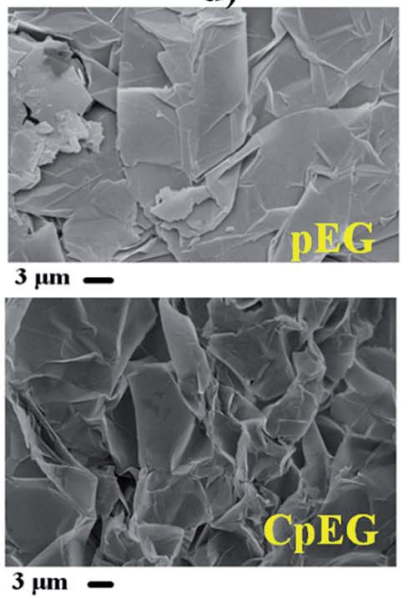

Fig. 1 Comparative characterizations of the pEG and CpEG samples: (a) TGA scans under air atmosphere. (b) X-ray diffraction patterns. (c) MicroRaman spectra recorded at the edge of the basal plane. (d) Scanning Electron Microscopy (SEM) images.

shows a more fluffy morphology consistent with a higher percentage of EG.

Table 1 shows the results of the elemental analysis of the graphitic samples. They highlight an oxygen content of $0.4 \mathrm{wt} \%$ for the sample pEG and $8.5 \mathrm{wt} \%$ for the sample CpEG. Sample pEG appears to be the sample with the smallest number of functional groups, in fact the value of the ratio $\mathrm{C} / \mathrm{O}$ is the highest one.

Fig. 2 shows the FTIR spectra of PEG and CpEG together with their appearance.

The visual observation which refers to the same amount of nanofiller (0.026 grams) gives an idea of the difference in volume between the samples pEG and CpEG. Although the difference in the exfoliation degree is quite small (7\%), it associated to the volume is very high (more than double). This

Table 1 Results of elemental analysis on anhydrous samples of $\mathrm{pEG}$ and $\mathrm{CPEG}$

Elemental composition (\% wt)

\begin{tabular}{llllllr}
\cline { 2 - 6 } Sample & $\mathrm{C}$ & $\mathrm{H}$ & $\mathrm{N}$ & $\mathrm{O}$ & $\mathrm{S}$ & $\mathrm{C} / \mathrm{O}$ \\
\hline pEG & 99.5 & 0.1 & 0.0 & 0.4 & 0.0 & 248.7 \\
CpEG & 91.3 & 0.2 & 0.0 & 8.5 & 0.0 & 10.7
\end{tabular}

result is not surprising for an investigation involving the nanofiller volume which is strongly affected by treatments acting at nano-scale level.

FTIR spectra of PEG and CpEG samples show the presence of oxygenated functional groups (mainly carboxylic, hydroxyl and epoxide), whose concentration is slightly lower in the pEG sample. The presence of different type of oxygen functionalities was confirmed by the bands at $3430 \mathrm{~cm}^{-1}(\mathrm{O}-\mathrm{H}$ stretching vibrations), at $1730-1740 \mathrm{~cm}^{-1}$ (stretching vibrations from $\mathrm{C}=\mathrm{O}$ ), at $1627-1635 \mathrm{~cm}^{-1}$ (skeletal vibrations from unoxidized graphitic domains), at $1220 \mathrm{~cm}^{-1}$ (C-OH stretching vibrations).

The samples pEG and CpEG show a quite similar profile of the bands except for the profile of the peaks between 1000 and $1300 \mathrm{~cm}^{-1}$ (in this spectral range, the profile of CpEG sample is more similar to the spectrum of SL graphene - see ESI $†$ ). In fact in CPEG sample we can observe the more intense peak at $1115 \mathrm{~cm}^{-1}$. This peak is most likely due to the presence of O-C-C stretching of aromatic esters. In fact, it is worth noting that $\mathrm{C}-\mathrm{C}-$ $\mathrm{O}$, and $\mathrm{O}-\mathrm{C}-\mathrm{C}$ stretches for aromatic ester fall into different ranges than saturated esters. ${ }^{27}$ In particular, the conjugation modifies the wavenumbers of the ester signals and the $\mathrm{O}-\mathrm{C}-\mathrm{C}$ stretch of aromatic esters falls between 1130 and $1100 \mathrm{~cm}^{-1}$.

In light of these considerations, we can aspect that, in phase of preparation of the filled samples, same of the -OH groups of carboxyl acids condense with - $\mathrm{OH}$ hydroxyl groups to form 

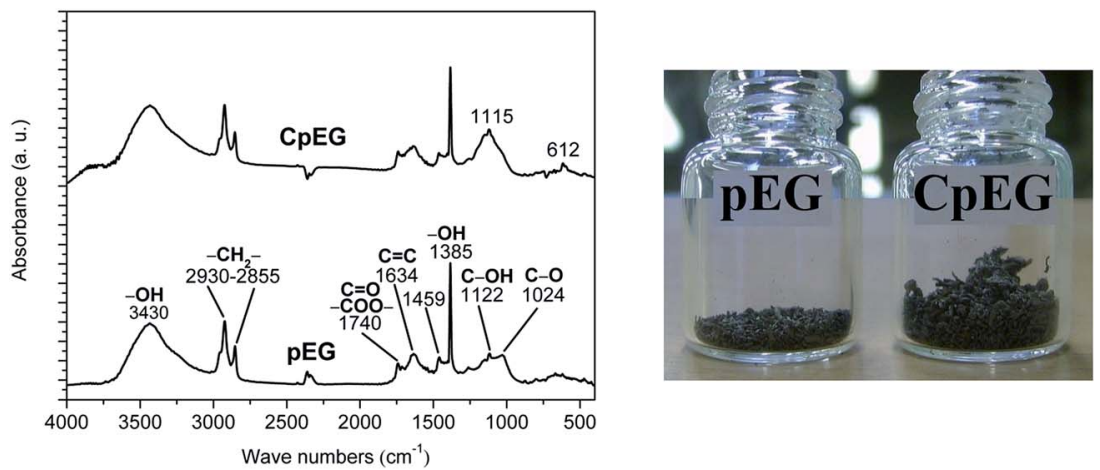

Fig. 2 FTIR spectra of the pEG and CpEG (on the left) and visual observation of pEG and CpEG samples characterized by the same weight (on the right).

aromatic ester groups according with the picture of the schematic arrangement (d) of Fig. 5 described in the next section.

It is worth noting that carboxyl groups are expected to be mainly located at the edge of graphene layers or graphitic blocks; ${ }^{28}$ then the previous results together with the elemental analysis highlight that sample CPEG is characterized by the higher concentration of carboxylated groups at the edge of graphene layers or graphitic blocks, which can create selfassembly structures through intermolecular hydrogen bonding that in some regions can also form aromatic ester groups bonding different graphene layers/blocks. In the case of SL graphene, due to the absence of graphitic blocks, functional groups attached to the layers may irreversibly crumple on them self, losing the planarity (see ESI $\dagger$ ).

\subsection{Characterization of the nanocomposites}

The polymer used for the matrix material was a mixture of tetraglycidylmethylene dianiline (TGMDA) and 1,4-butanedioldiglycidylether (BDE). This particular epoxy formulation has proven to be very effective for improving nanofiller dispersion due to a decrease in the viscosity, ${ }^{29-31}$ and to reduce the moisture content which is a very critical point for structural epoxy resins. ${ }^{32,33}$

The powders of pEG and CpEG samples were embedded inside the epoxy mixture which was solidified using the experimental procedure described in the section "Experimental".

Fig. 3 shows (a) the X-ray diffraction patterns of the unfilled epoxy mixture TBD, and the nanofilled epoxy composites TBD-pEG 2\% wt, TBD-CpEG 1.8\% wt and (b) TEM images of the nanofilled epoxy composite at $1.8 \mathrm{wt} \%$ of CpEG (on the left) and $2 \mathrm{wt} \%$ of pEG (on the right).

The X-ray diffraction patterns of nanofilled epoxy composite with $2 \mathrm{wt} \%$ of pEG and $1.8 \mathrm{wt} \%$ of CpEG show the presence, in both nanocomposites, of intense 002 peaks as well as of weak 112 peaks, indicating the maintenance in the composite of the hexagonal graphite stacks (with a correlation length in the range 40-45 $\mathrm{nm}$ ) of the considered fillers. The effect of the processing conditions on structural organization of pEG and CpEG is discussed in detail in the section ESI. $\dagger$
Fig. 3(b) shows TEM images of the two epoxy-based composites. The nanoparticles in the resin consist of small stacks of graphene that are from 1 to about $16 \mathrm{~nm}$ thick, with diameters ranging from sub-micrometer to hundreds of $\mu \mathrm{m}$. Multilayer graphene stacks are observable in both the samples. In particular, thin arrows indicate the edges of the individual layers and the bold arrow points out a folded off-plane fragment of layer. From the high magnification of the TEM image (top side, on the right), the thickness of block of pEG can be estimated to be about $16 \mathrm{~nm}$. TEM images of pEG powder show the crumpled morphology of the few layer of graphene with wrinkled and disordered sheet-like structure (bottom side, on the right). From the folded edge of exfoliated graphite nanoplatelets in the TEM images, the thickness can be estimated to be several $\mathrm{nm}$. A very relevant morphological feature is shown by the resin filled with CpEG sample (see on the left of the TEM images). Graphene sheets and thin graphite blocks seem also selfassembled leading to extended architectures assembled along the sample (see red ellipses).

A very interesting morphological feature of CpEG sample is also clearly visible in AFM images of the fracture surface of the nanofilled epoxy composite at loading concentration of $1.8 \%$ by weight of nanofiller (see Fig. 4(a)-(b)). The AFM images were collected on etched samples to partially remove the resin surrounding the graphene sheets and to better observe the distribution of the filler inside the epoxy matrix.

In Fig. 4(a) and (b), thin graphite blocks seem assembled along the sample (see red ellipses in Fig. 4(a)).

The distance between graphitic stacks (see Fig. 4(b)) is approximatively between 5-10 $\mathrm{nm}$.

This assembling effect seems less visible in the AFM images of the fracture surface of the nanofilled epoxy composite even at the high loading concentration of $3 \%$ by weight of pEG (see Fig. 4(c))

Also in this case, it is possible to observe folded graphene sheets resembling the draping of a textile with parallel preferential orientation of graphene layers/blocks (see in phase image - blue arrows); but in contrast with the previous AFM images, the graphitic stacks appear thicker, separated by a larger gap and randomly distributed. 
a)

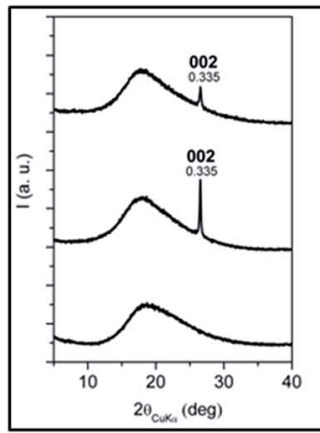

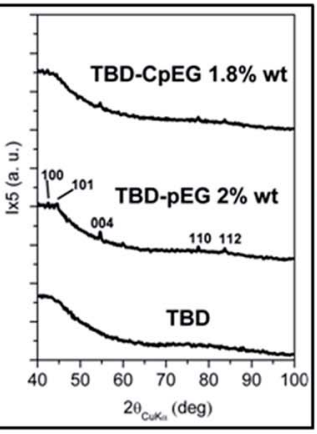

Very thin blocks of carboxylated graphite, although well separated from each other, constitute SelfAssembly structures extended through the sample b)

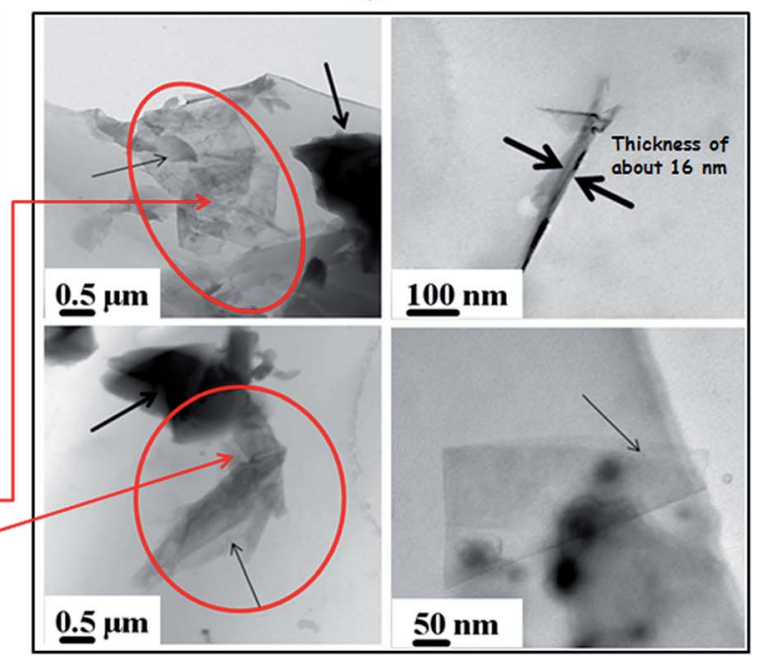

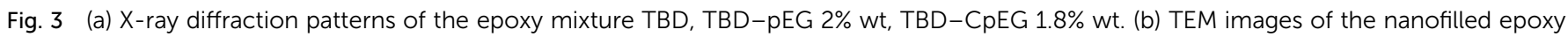
composite at $1.8 \mathrm{wt} \%$ of CpEG (on the left) and 2 wt\% of pEG (on the right).

Fig. 5 shows (a) the electrical conductivity of the resins filled with PEG and CPEG as a function of the filler concentration and ( $b$ and c) the results of dynamic mechanical properties related to the storage modulus (b) and loss factor $(\tan \delta$ ) (c) of the sample TBD-2\% pEG, and sample TBD-1.8\% CpEG, and (d) the schematic arrangement of edge-carboxylated graphene particles.

The effectiveness of a nanofiller and specifically of graphenebased materials can be evaluated by considering the achievement of a low percolation threshold (EPT) and high conductivity at low loading percentage.

From Fig. 5(a) it can be noted that the adopted epoxy matrix is characterized by a volume conductivity at room temperature of about $6 \times 10^{-14} \mathrm{~S} \mathrm{~m}^{-1}$. The introduction of graphite-based fillers significantly affects the electrical behavior of the composite leading to an abrupt transition from an insulating to a conducting behavior. The conductivity, depending on the amount of exfoliated graphite characterizing the filler, shows an increase of more than five order of magnitude compared to that of the neat resin. More specifically, the DC conductivity at the very low loading of $1.8 \% \mathrm{wt}$ for CpEG system, reaches the value of about $0.096 \mathrm{~S} \mathrm{~m}^{-1}$ whereas it is of the order of few $\mathrm{pS}$ for pEG. This last system achieves comparable values of the conductivity for more than double filler concentration (around $4 \% \mathrm{wt}$ ). In addition, the remarkable variation in the value of EPT can be hardly attributable exclusively to the variation of EG percentage between the two samples. In fact, such parameter decreases (i.e. about $3 \%$ for samples pEG and in the range $0.025-0.1 \%$ for samples CpEG) reaching values, which to the best of the authors knowledge, have never been reported in literature for epoxy resin loaded with this type of filler. These low EPTs are instead observed when using mono-dimensional carbon based nanoparticles, such as carbon nanotubes (CNTs) or carbon nanofibers (CNFs). ${ }^{31-34}$

The observed decrease in EPT depends, most probably, on a contribution of several synergic factors, for example among them the higher concentration of carboxylated groups at the edge of graphene sheets. Such a conjectured mechanism, which is prevalently observed in TEM images of CPEG, may allow attractive intermolecular bonding between graphitic nanoparticles, which in turn favors the formation of more efficient conducting networks in this type of nanocomposites.

This hypothesis seems to be strongly proven by TGA curves of the fillers which indicate an amount of carboxylated groups of about $10 \mathrm{wt} \%$ for the sample CpEG. On the other hand, FTIR investigation indicates that some of the - $\mathrm{OH}$ groups of carboxyl acids condense with $-\mathrm{OH}$ hydroxyl groups to form aromatic ester groups. This last mechanism is also active during the preparation of the nanocomposites and together with the intramolecular H-bonds may be responsible of self-assembly structures which favor the electrical conductive paths.

It is worth noting that the carboxylic acid moiety is considered to be a highly polar organic functional group. This polarity results from the presence of a strongly polarized carbonyl $(\mathrm{C}=\mathrm{O})$ group and hydroxyl $(\mathrm{O}-\mathrm{H})$ group. The oxygen is a relatively electronegative atom and when covalently bound to carbon and particularly hydrogen, a strong permanent dipole is created. In the case of carboxylic acids, the $\mathrm{O}-\mathrm{H}$ group is even more strongly polarized than the $\mathrm{O}-\mathrm{H}$ group of alcohols due to the presence of the adjacent carbonyl moiety.

These structural features enhance dipole strength and are responsible for attractions between permanent dipole-dipole interactions and $\mathrm{H}$-bonds. Hence, the dipoles present in carboxylic acids allow these compounds to participate in energetically favorable hydrogen bonding (H-bonding) interactions also with the polar groups of the resin network. This specific last mechanism should favor a more efficient load transfer between matrix and filler causing a strong mechanical reinforcement.

The energy associated with the dipoles present in carboxylic acids on graphitic layers could be directly reflected by mechanical properties. In light of this last consideration, 

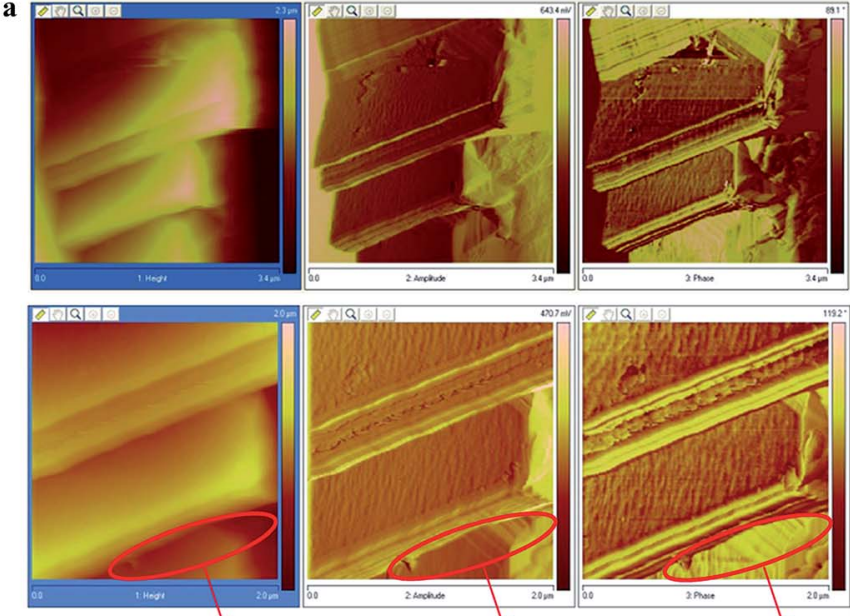

$\downarrow$

thin graphite blocks seem assembled along the sample
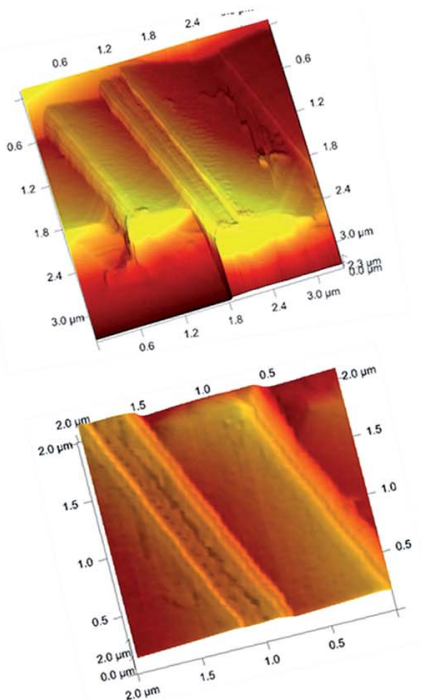

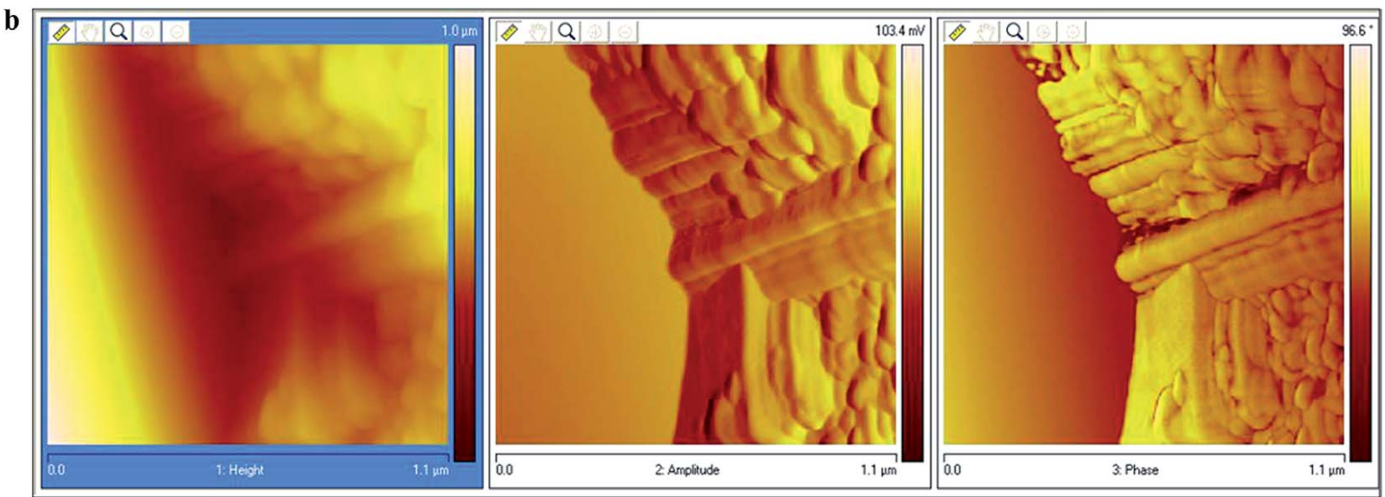

c
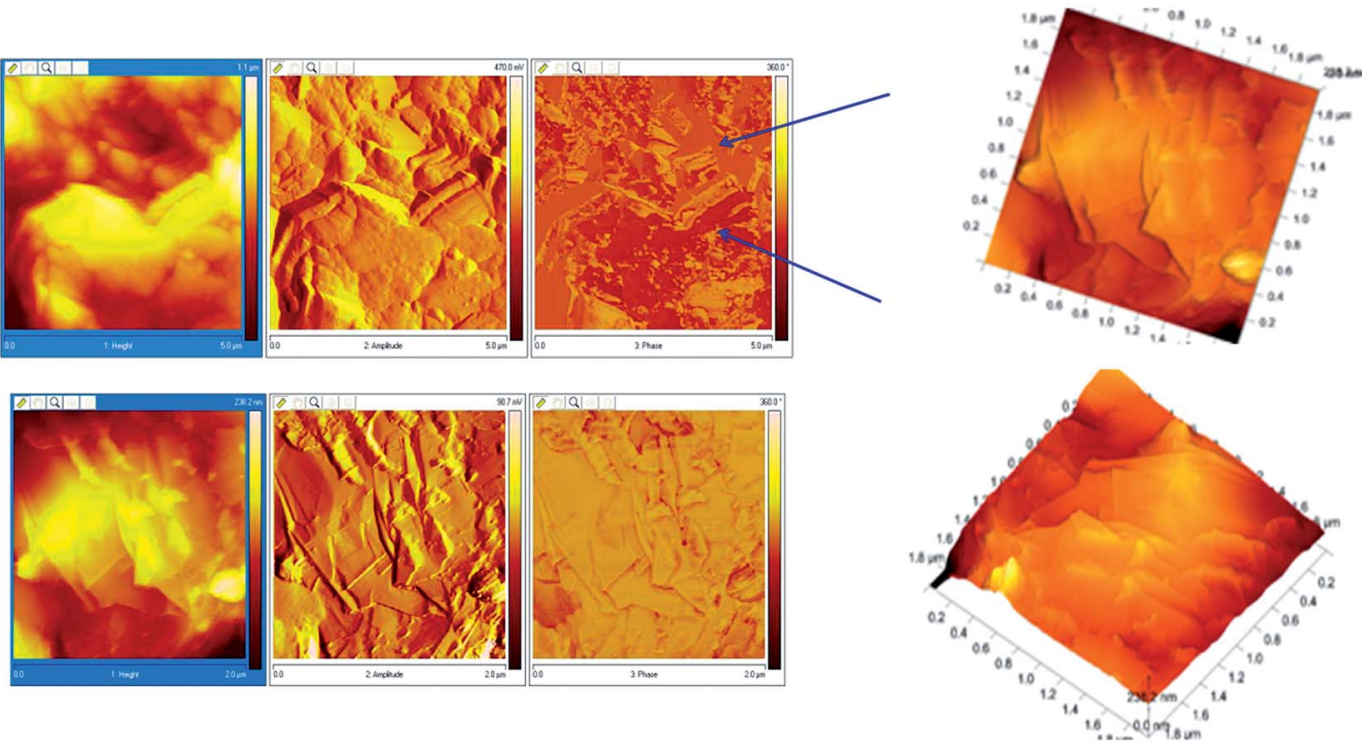

Fig. 4 (a) AFM images of the fracture surface of the nanofilled epoxy composite at loading concentration of $1.8 \%$ by weight of CpEG. (b) AFM images of the fracture surface of the nanofilled epoxy composite at loading concentration of $1.8 \%$ by weight of CpEG. (c) AFM images of the fracture surface of the nanofilled epoxy composite at loading concentration of $3 \%$ by weight of $p E G$. 


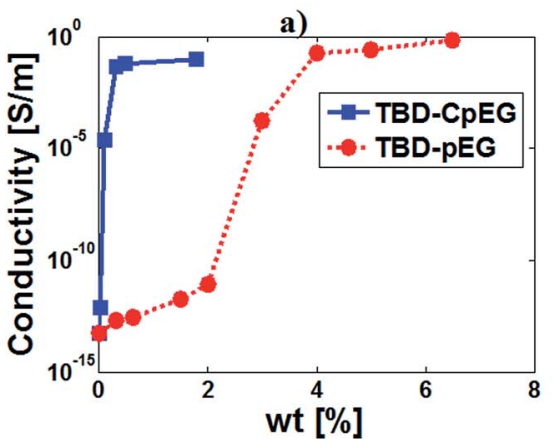

c)

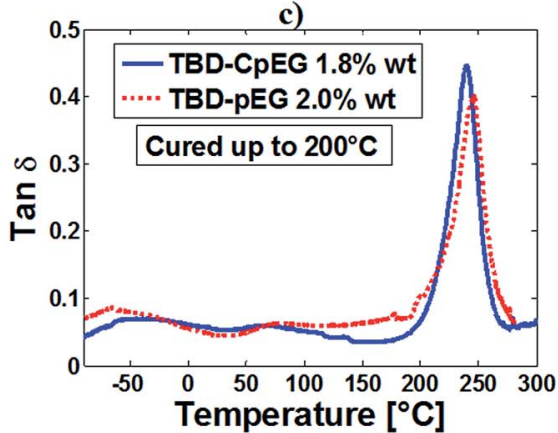

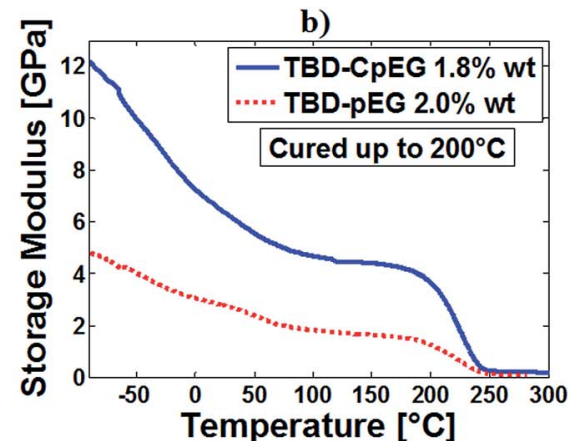

d)

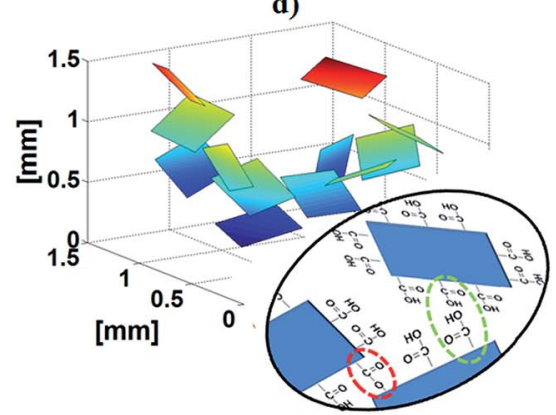

Fig. 5 Electrical conductivity (a), storage modulus (b) and loss factor (tan $\delta$ ) (c) of sample TBD-CpEG 1.8\% wt and TBD-pEG 2.0\% wt, schematic arrangement of edge-carboxylated graphene particles (d) (colors are not indicative of specific properties).

dynamic mechanical tests were performed to verify this hypothesis.

Fig. 5(b) shows a strong increase in the storage modulus in a wide range of temperatures, in particular in the range -90 to $210{ }^{\circ} \mathrm{C}$ for the TBD $+1.8 \%$ CpEG sample with respect to the TBD $+2 \%$ pEG sample and TBD sample (not reported here). No significant change is instead observed in the glass transition temperature $\left(T_{\mathrm{g}}\right)$ which is between 240 and $280^{\circ} \mathrm{C}$ for both the formulations (Fig. 5(c)). This strong reinforcement in the storage modulus of the samples with CPEG embedded in the matrix was found also with different composition of the hardener agent. At room temperature, for a concentration of CpEG of $1.0 \mathrm{wt} \%$ an increase of $80 \%$ was found in the storage modulus.

It is interesting to note that, while the strong increase in the electrical conductivity is easily obtainable with conductive nanofillers $1 \mathrm{D},{ }^{32,34}$ such an increase in the elastic modulus has never been obtained with mono-dimensional conductive nanofillers.

Data and discussion on the properties of epoxy/graphene nanocomposites highlight the dominant role of the exfoliation degree and edge-carboxylated graphite layers to design lightweight materials, characterized by both low electrical percolation thresholds and improved mechanical properties. So far, this synergy has been a problematic challenge to achieve because nanofiller functionalization can provide superb polymer-particle interactions which are very effective for improving the mechanical characteristics, but at same time may worsen electrical conductivity of the nanofilled polymer. In the nanocomposites filled with CpEG nanoparticles, we get a significant advancement on these two fronts simultaneously. In fact, as for other nanostructured forms of carbons, ${ }^{34}$ the presence of carboxylated groups determines an increase in the storage modulus. This increase is very consistent also due to selfassembly mechanisms determined by attractive interactions mainly based on hydrogen bonding between edge-carboxylated graphitic blocks present in major amount on the edges of CpEG nanoparticles (see green ellipse of Fig. 5(d)).

FTIR spectra previous discussed highlight that edgecarboxylated graphitic blocks can create self-assembly structures that in some regions can also form aromatic ester groups bonding different graphene layers/blocks, as illustrated in Fig. 5(d) (see red ellipse). The self-assembly structures represent an ideal condition to bring together the conductive graphitic blocks along the percolation paths.

Edge-selective carboxylated nanosheets were already prepared by other authors. ${ }^{23}$ Electrical conductivity of $1.1 \mathrm{~S} \mathrm{~m}^{-1}$ was found for ECG pellets alone (not embedded inside the polymeric matrix), whereas we have found similar values for nanocomposites with very low filler concentrations. This is a remarkable result especially if the excellent mechanical performances achieved at once very high value in $T_{g}$, strong increase in the storage modulus, high thermal stability (see ESI $\dagger$ ) are considered.

A detailed comparison on the physical characterizations of the same epoxy resin filled with SL graphene sheets is available in the section "ESI". $\dagger$

\section{Conclusion}

In this study, two sample types characterized by different degree of graphite exfoliation (partially exfoliated graphite pEG and CpEG) and functionalization (carboxylated groups in CpEG) 
were made. Then nanocomposites were prepared by embedding in the resin PEG and CpEG graphite particles.

It has been found that the percolation threshold reduces from $3 \mathrm{wt} \%$ down to $0.3 \mathrm{wt} \%$, as the carboxylated group content increases up to $10 \mathrm{wt} \%$. Self-assembly of blocks due to attractive interactions between edge-carboxylated graphene particles favors the electrical percolative paths. Edge-carboxylated nanosheets also increase the nanofiller/epoxy matrix interaction, determining a relevant reinforcement in the storage modulus. A comparative study was made between published data and our results. It shows that edge structures of graphitic blocks can be controlled to develop new generation of polymers with enhanced structural and electrical properties.

The surface chemistry of thin graphitic edges can be tuned at nanoscale level, to pave the way towards an effective strategy to overcome drawbacks related to the application of graphenebased materials.

A sound choice of the nanofiller nature allows to drive the changes in the nanocomposite properties towards the set goals. In this study, it has been found that the functionalization help in providing a better polymer filler interface. This interface had help in enhancing both electrical and mechanical properties. These tangible results were obtained because of strong interactions between nanofiller and epoxy matrix and self-assembly structures in this kind of matrix. Work is still in progress, but a giant step towards understanding the electrical and mechanical behaviour of in-bulk resins filled with bi-dimensional shaped carbon forms was taken. A further relevant aspect to study is the effects of graphite exfoliation and functionalization on a non-polar matrix (such as polyethylene). In order to provide sound conclusions for this type of matrix, a detailed study is required which takes into account also the different experimental procedures for preparing the nanocomposites which are deemed to have a strong influence on the observed properties. The results of this study will be presented in a future paper.

\section{Experimental}

\subsection{Materials}

Two samples of partially exfoliated graphite (pEG) and carboxylated partially exfoliated graphite (CpEG), differing for the content of carboxylated groups, were prepared with the aim to achieve consistent comprehension about the properties of resins filled with graphene-based nanoparticles.

Graphene-based nanoparticles pEG and CPEG are characterized by two different degrees of amorphous phase $\left(\chi_{\mathrm{a}}\right)$ : $56 \%$ (pEG) and 60\% (CpEG).

The elementary analysis of the graphitic samples highlighted an oxygen content of $0.4 \mathrm{wt} \%$ for the sample pEG and $8.5 \mathrm{wt} \%$ for the sample CpEG. The sample PEG and CPEG were prepared as follows: a mixture containing nitric and sulphuric acid and natural graphite was used. After $24 \mathrm{~h}$ of reaction, intercalation within graphene sheets took place to form intercalated graphite compound. Then the mixture was filtered, washed with water, and dried in an oven at low temperatures. The intercalated graphite compound was subjected to sudden heat treatment temperature of $900{ }^{\circ} \mathrm{C}$ and rapid expansion then occurred. The expansion ratio was as high as 300 times. Change in the degree of exfoliation was obtained by varying the resident time in the fluidized bed as the time increases, the trapped intercalate and/or gases would have a second the chance to escape causing further expansion and exfoliation. The considered filler has a two dimensional (2D) predominant shape and it is obtained with an exfoliation procedure from natural graphite, that leads to obtain $2 \mathrm{D}$ conductive particles with an average diameter of $500 \mu \mathrm{m}$.

The epoxy matrix was prepared by mixing a tetrafunctional precursor with a reactive diluent which allows to reduce the moisture content and to facilitate the dispersion step of nanofillers. ${ }^{32}$

All the mixtures were cured by a two-stage curing cycles: a first isothermal stage was carried out at the lower temperature of $125^{\circ} \mathrm{C}$ for 1 hour and the second isothermal stage at higher temperatures up to $200{ }^{\circ} \mathrm{C}$ for 3 hours.

\subsection{Characterization}

Scanning electron microscope micrographs were obtained with a field emission Scanning Electron Microscopy (SEM) apparatus (JSM-6700F, JEOL) instrument operating at $3 \mathrm{kV}$. Some of the nanocomposites section were cut from the solid samples by a sledge microtome. These slices were etched before the observation by SEM. The etching reagent was prepared by stirring $1.0 \mathrm{~g}$ potassium permanganate in a solution mixture of $95 \mathrm{~mL}$ sulfuric acid (95-97\%) and $48 \mathrm{~mL}$ orthophosphoric acid (85\%). The filled resins were immersed into the fresh etching reagent at room temperature and held under agitation for 36 hours. Subsequent washings were done using a cold mixture of 2 parts by volume of concentrated sulfuric acid and 7 parts of water. Afterwards the samples were washed again with $30 \%$ aqueous hydrogen peroxide to remove any manganese dioxide. The samples were finally washed with distilled water and kept under vacuum for 5 days.

The measurements of the dc volume conductivity were performed by using disk-shaped specimens of about $2 \mathrm{~mm}$ thickness and $50 \mathrm{~mm}$ diameter, the measurement electrode is circular with a diameter of about $22 \mathrm{~mm}$. The measurement system, remotely controlled by the software LABVIEW $\AA$, is composed of a suitable shielded cell with temperature control, of multimeter Keithley 6517A with function of voltage generator (max. $\pm 1000 \mathrm{~V}$ ) and voltmeter (max. $\pm 200 \mathrm{~V}$ ) and the ammeter HP34401A (min. current $0.1 \mu \mathrm{A}$ ) for samples above the percolation threshold. For ones below the percolation threshold the system is composed only of multimeter Keithley 6517A with function of voltage generator ( $\max . \pm 1000 \mathrm{~V}$ ) and pico-ammeter (min. current $0.1 \mathrm{fA}$ ).

Dynamic mechanical properties of the samples were performed with a dynamic mechanical thermo-analyzer (Tritec 2000 DMA-Triton Technology). Solid samples with dimensions $2 \times 10 \times 35 \mathrm{~mm}^{3}$ were tested by applying a variable flexural deformation in three points bending mode. The displacement amplitude was set to $0.03 \mathrm{~mm}$, whereas the measurements were performed at the frequency of $1 \mathrm{~Hz}$. The range of temperature was from $-90{ }^{\circ} \mathrm{C}$ to $315^{\circ} \mathrm{C}$ at the scanning rate of $3{ }^{\circ} \mathrm{C} \mathrm{min}^{-1}$. 
Wide-angle X-ray diffraction (WAXD) patterns in reflection were obtained by an automatic Bruker D8 Advance diffractometer operating at $35 \mathrm{kV}$ and $40 \mathrm{~mA}$. The nickel filtered $\mathrm{Cu}-\mathrm{K} \alpha$ radiation $(1.5418 \AA)$ was used. $d$-spacings were calculated using Bragg's law.

The correlation lengths $(D)$ of the graphitic nanofiller were determined using Scherrer's equation (eqn (1) $)^{35}$

$$
D=\frac{K \lambda}{\beta \cos \theta}
$$

where $K$ is the Scherrer constant, assumed $=1, \lambda$ is the wavelength of the incident $\mathrm{X}$-rays, $\theta$ is the diffraction angle and $\beta$ is the corrected integral breadth, obtained by subtracting the instrumental broadening of the closest silicon reflection from the observed integral breadths, as follows: $\beta=\beta_{\text {obs }}-\beta_{\text {inst. }} \cdot \beta_{\text {obs }}$ were determined through a fit with a Lorentzian function of the intensity corrected diffraction patterns, adopting the procedure described by Iwashita et al. ${ }^{36}$

The instrumental broadening ( $\left.\beta_{\text {inst }}\right)$ was also determined through a fitting of Lorentzian function to line profiles of a standard silicon powder 325 mesh (99\%). The degree of crystallinity $\left(\chi_{\mathrm{c}}\right)$ of the graphitic nanofiller was evaluated from X-ray diffraction data, applying the standard procedure of resolving the diffraction pattern into two areas, $A_{\mathrm{c}}$ and $A_{\mathrm{a}}$, that can be taken as proportional to the crystalline and the amorphous fraction of EG, respectively, and calculated, for the $2 \theta$ range $15-40^{\circ}$, using the equation (eqn (2))

$$
\chi_{\mathrm{c}}=\frac{A_{\mathrm{c}}}{A_{\mathrm{c}}+A_{\mathrm{a}}} \times 100
$$

according to the classical Hermans-Weidinger method. ${ }^{37}$

Raman spectra were obtained at room temperature with a micro-Raman spectrometer Renishaw inVia with a $514 \mathrm{~nm}$ excitation wavelength (laser power $30 \mathrm{~mW}$ ) in the range (100-3000 $\left.\mathrm{cm}^{-1}\right)$. In order to separate the individual peaks in the case of unresolved, multicomponent bands, a curve resolving algorithm was applied, based on the LevenbergMarquardt method; ${ }^{26}$ to reduce the number of adjustable parameters and to insure the uniqueness of the result, the baseline, the band shape and the number of components were fixed. The minimum number of components was evaluated by visual in section on the basis of abrupt changes of slope of the experimental line-shape. The program was then allowed to calculate, by a non-linear curve fitting of data, the height, the full width al half height (FWHH) and the position of the individual components. The peak function was a mixed GaussLorentz line shape of the form: ${ }^{38}$

$$
f(x)=(1-L) H \exp \left[-\frac{x-x_{0}}{w}[4 \ln 2]\right]+L \frac{H}{4\left(\frac{x-x_{0}}{w}\right)+1}
$$

where $x_{0}=$ peak position; $H=$ peak height; $w=$ FWHH; $L=$ fraction of Lorentz character.

Elemental analysis was performed with a Thermo FlashEA 1112 Series CHNS-O analyzer, after pre-treating samples in an oven at $100{ }^{\circ} \mathrm{C}$ for $12 \mathrm{~h}$.

\section{Acknowledgements}

The research leading to these results has received funding from the European Union's Seventh Framework Programme for research, technological development and demonstration under Grant Agreement no. 313978.

\section{References}

1 I. Kranauskaite, J. Macutkevic, P. Kuzhir, N. Volynets, A. Paddubskaya, D. Bychanok, S. Maksimenko, J. Banys, R. Juskenas, S. Bistarelli, A. Cataldo, F. Micciulla, S. Bellucci, V. Fierro and A. Celzard, Dielectric properties of graphite-based epoxy composites, Phys. Status Solidi A, 2014, 211, 1623-1633.

2 W. Lu, J. Weng, D. Wu, C. Wua and G. Chen, Epoxy Resin/ Graphite Electrically Conductive Nanosheet Nanocomposite, Mater. Manuf. Processes, 2006, 21, 167-171.

3 B. Debelak and K. Lafdi, Use of exfoliated graphite filler to enhance polymer physical properties, Carbon, 2007, 45, 1727-1734.

4 R. Sengupta, M. Bhattacharya, S. Bandyopadhyay and A. K. Bhowmick, A review on the mechanical and electrical properties of graphite and modified graphite reinforced polymer composites, Prog. Polym. Sci., 2011, 36, 638-670.

5 S. Ganguli, A. K. Roy and D. P. Anderson, Improved thermal conductivity for chemically functionalized exfoliated graphite/epoxy composites, Carbon, 2008, 46, 806-817.

6 A. Dabrowska, S. Bellucci, A. Cataldo, F. Micciulla and A. Huczko, Nanocomposites of epoxy resin with graphene nanoplates and exfoliated graphite: Synthesis and electrical properties, Phys. Status Solidi B, 2014, 251, 2599-2602.

7 P. Kuzhir, A. Paddubskaya, A. Plyushch, N. Volynets, S. Maksimenko, J. Macutkevic, I. Kranauskaite, J. Banys, E. Ivanov, R. Kotsilkova, A. Celzard, V. Fierro, J. Zicans, T. Ivanova, R. Merijs Meri, I. Bochkov, A. Cataldo, F. Micciulla, S. Bellucci and P. Lambin, Epoxy composites filled with high surface area-carbon fillers: Optimization of electromagnetic shielding, electrical, mechanical, and thermal properties, J. Appl. Phys., 2013, 114, 164304.

8 S. Stankovich, D. A. Dikin, G. H. B. Dommet, K. M. Kohlhaas, E. L. Zimney, E. A. Stach, R. D. Piner, T. N. SonBinh and R. S. Ruoff, Graphene-based composite materials, Nature, 2006, 442, 282-286.

9 W. Xia, C. Jin, S. Kundu and M. Muhler, A highly efficient gas-phase route for the oxygen functionalization of carbon nanotubes based on nitric acid vapor, Carbon, 2009, 47, 919-922.

10 H. Pang, T. Chen, G. Zhang, B. Zeng and Z. M. Li, An electrically conducting polymer/graphene composite with a very low percolation threshold, Mater. Lett., 2010, 64, 22262229.

$11 \mathrm{M}$. Yoonessi and J. R. Gaier, Highly Conductive Multifunctional Graphene Polycarbonate Nanocomposites, ACS Nano, 2010, 4, 7211-7220.

12 Y. T. Liu, X. M. Xie and X. Y. Ye, High-concentration organic solutions of poly(styrene-co-butadiene-co-styrene)-modified 
graphene sheets exfoliated from graphite, Carbon, 2011, 49, 3529-3537.

13 V. H. Pham, T. V. Cuong, T. T. Dang, S. H. Hur, B. S. Kong, E. J. Kim, E. W. Shin and J. S. Chung, Superior conductive polystyrene - chemically converted graphene nanocomposite, J. Mater. Chem., 2011, 21, 11312-11316.

14 Y. Zhan, M. Lavorgna, G. Buonocore and H. Xia, Enhancing electrical conductivity of rubber composites by constructing interconnected network of self-assembled grapheme with latex mixing, J. Mater. Chem., 2012, 22, 10464-10468.

15 N. Yousefi, M. M. Gudarzi, Q. Zheng, S. H. Aboutalebi, F. Sharif and J. K. Kim, Self-alignment and high electrical conductivity of ultralarge graphene oxide-polyurethane nanocomposites, J. Mater. Chem., 2012, 22, 12709-12717.

16 B. Wang, H. Li, L. Li, P. Chen, Z. Wang and Q. Gu, Electrostatic adsorption method for preparing electrically conducting ultrahigh molecular weight polyethylene/ graphene nanosheets composites with a segregated network, Compos. Sci. Technol., 2013, 89, 180-185.

17 N. Yousefi, X. Lin, Q. Zheng, X. Shen, J. R. Pothnis, J. Jia, E. Zussman and J. K. Kim, Simultaneous in situ reduction, self-alignment and covalent bonding in graphene oxide/ epoxy composites, Carbon, 2013, 59, 406-417.

18 A. S. Wajid, H. S. T. Ahmed, S. Das, F. Irin, A. F. Jankowski and M. J. Green, High-Performance Pristine Graphene/ Epoxy Composites With Enhanced Mechanical and Electrical Properties, Macromol. Mater. Eng., 2013, 298, 339-347.

19 H. Wu, W. Zhao, H. Hu and G. Chen, One-step in situ ball milling synthesis of polymer-functionalized graphene nanocomposites, J. Mater. Chem., 2011, 21, 8626-8632.

20 J. Yu, X. Huang, C. Wu and P. Jiang, Permittivity, thermal conductivity and thermal stability of poly(vinylidene fluoride)/graphene nanocomposites, IEEE Trans. Dielectr. Electr. Insul., 2011, 18, 478-484.

21 Y. Li, J. Zhu, S. Wei, J. Ryu, L. Sun and Z. Guo, Poly(propylene)/Graphene Nanoplatelet Nanocomposites: Melt Rheological Behavior and Thermal, Electrical, and Electronic Properties, Macromol. Chem. Phys., 2011, 212, 1951-1959.

22 A. Guimont, E. Beyou, P. Alcouffe, P. Cassagnau, A. Serghei, G. Martin and P. Sonntag, Pentadecane functionalized graphite oxide sheets as a tool for the preparation of electrical conductive polyethylene/graphite oxide composites, Polymer, 2014, 55, 22-28.

23 I. Y. Jeon, Y. R. Shin, G. J. Sohn, H. J. Choi, S. Y Bae, J. Mahmood, S. M. Jung, J. M. Seo, M. J. Kim, D. W. Chang, L. Dai and J. B. Baek, Edge-carboxylated graphene nanosheets via ball milling, Proc. Natl. Acad. Sci. U. S. A., 2012, 109, 5588-5593.

24 R. P. Vidano and D. B. Fischbach, Observation of Raman band shifting with excitation wavelength for carbons and graphites, Solid State Commun., 1981, 39, 341-344.
25 A. C. Ferrari, J. C. Meyer, V. Scardaci, C. Casiraghi, M. Lazzeri, F. Mauri, S. Piscanec, D. Jiang, K. S. Novoselov, S. Roth and A. K. Geim, Raman Spectrum of Graphene and Graphene Layers, Phys. Rev. Lett., 2006, 97, 187401-187404.

26 D. W. Marquardt, An Algorithm for Least-Squares Estimation of Nonlinear Parameters, J. Soc. Ind. Appl. Math., 1963, 11, 431-441.

27 B. C. Smith, Infrared Spectral Interpretation: A Systematic Approach, CRC Press, December 10, 1998, p. 288, ISBN 9780849324635, printed in United States of America.

28 S. Gadipelli and Z. X. Guo, Graphene-based materials: Synthesis and gas sorption, storage and separation, Prog. Mater. Sci., 2015, 69, 1-60.

29 L. Guadagno, M. Raimondo, K. Lafdi, A. Fierro, S. Rosolia and M. R. Nobile, Influence of nanofiller morphology on the viscoelastic properties of CNF/epoxy resins, AIP Conf. Proc., 2014, 1599, 386-389.

30 M. R. Nobile, M. Raimondo, K. Lafdi, A. Fierro, S. Rosolia and L. Guadagno, Relationships Between Nanofiller Morphology and Viscoelastic Properties in CNF/Epoxy Resins, Polym. Compos., 2015, DOI: 10.1002/pc.23362, article in press.

31 L. Guadagno, M. Raimondo, U. Vietri, L. Vertuccio, G. Barra, B. De Vivo, P. Lamberti, G. Spinelli, V. Tucci, R. Volponi, G. Cosentino and F. De Nicola, Effective Formulation and Processing of Nanofilled Carbon Fiber Reinforced Composites, RSC Adv., 2015, 5, 6033-6042.

32 L. Guadagno, M. Raimondo, V. Vittoria, L. Vertuccio, C. Naddeo, S. Russo, B. De Vivo, P. Lamberti, G. Spinelli and V. Tucci, Development of epoxy mixtures for application in aeronautics and aerospace, RSC Adv., 2014, 4, 15474-15488.

33 L. Guadagno, M. Raimondo, V. Vittoria, L. Vertuccio, C. Naddeo, P. Lamberti and V. Tucci, Italian Patent Resina epossidica con basso tenore di umidità No. TO2013A000926, 15 November 2013.

34 L. Guadagno, M. Raimondo, V. Vittoria, L. Vertuccio, K. Lafdi, B. De Vivo, P. Lamberti, G. Spinelli and V. Tucci, The role of carbon nanofiber defects on the electrical and mechanical properties of CNF-based resins, Nanotechnology, 2013, 24, 305704.

35 H. Tadokoro, Structure of Crystalline Polymers, John Wiley\& Sons, New York, 1979.

36 N. Iwashita, C. R. Park, H. Fujimoto, M. Shiraishi and M. Inagaki, Specification for a standard procedure of X-ray diffraction measurements on carbon materials, Carbon, 2004, 42, 701-714.

37 P. H. Hermans and A. Weidinger, On the determination of the crystalline fraction of polyethylenes from X-ray diffraction, Makromol. Chem., 1961, 44, 24-36.

38 W. F. Maddams, The Scope and Limitations of Curve Fitting, Appl. Spectrosc., 1980, 34, 245-267. 